\title{
Ambiguous Effects of Prolonged Dietary Supplementation with a Striatal-Enriched Protein Tyrosine Phosphatase Inhibitor, TC-2153, on a Rat Model of Sporadic Alzheimer's Disease
}

\author{
E. A. Rudnitskaya ${ }^{a}$, A. O. Burnyasheva ${ }^{a}$, T. A. Kozlova ${ }^{a}$, N. A. Muraleva ${ }^{a}$, D. V. Telegina ${ }^{a}$, \\ T. M. Khomenko ${ }^{b}$, K. P. Volcho ${ }^{b}$, N. F. Salakhutdinov ${ }^{b}$, and N. G. Kolosova ${ }^{a, 1}$ \\ ${ }^{a}$ Institute of Cytology and Genetics SB RAS, Novosibirsk, Russia \\ ${ }^{b}$ N.N. Vorozhtsov Novosibirsk Institute of Organic Chemistry SB RAS, Novosibirsk, Russia \\ Received March 19, 2021; revised March 24, 2021; accepted March 29, 2021
}

\begin{abstract}
Alzheimer's disease (AD) is the most common type of dementia and is currently incurable. After unsuccessful attempts to create drugs targeting the amyloid- $\beta$ pathway, a search for alternative approaches and treatments targeting nonamyloid AD pathologies is currently underway. One of them is inhibition of striatal-enriched protein tyrosine phosphatase (STEP) activity, which is increased in the prefrontal cortex of AD patients. Here we examined effects of prolonged treatment of OXYS rats which mimic key signs of sporadic AD with a STEP inhibitor, TC-2153, on the progression of signs of AD. TC-2153 had an ambiguous effect on the behavior of the animals: it significantly reduced the already low locomotor and exploratory activities and enhanced anxiety-related behavior in OXYS rats but improved their long-term memory in the Morris water maze. Moreover, TC-2153 had no effect on the accumulation of the amyloid- $\beta$ protein and on the STEP61 protein level; the latter in the cortex and hippocampus did not differ between OXYS rats and control Wistar rats. These results suggest that the effects of prolonged treatment with TC-2153 may be mediated by mechanisms not related to STEP. In particular, TC-2153 can act as a potential hydrogen sulfide donor and thus substantially affect redox homeostasis.
\end{abstract}

Keywords: Alzheimer's disease, striatal-enriched protein tyrosine phosphatase STEP, STEP inhibitor, synthetic compound TC-2153, OXYS rats

DOI: $10.1134 / \mathrm{S} 1819712421030090$

\section{INTRODUCTION}

Alzheimer's disease (AD) is a progressive incurable age-related neurodegenerative disorder in the elderly and the most prevalent cause of dementia [1,2]. AD is clinically characterized by memory deficits, changes in mood and personality, problems with communication, confusion about places, people and events, and impaired cognitive functions. Neuropathologically, $\mathrm{AD}$ is characterized by various neurological factors such as amyloid plaques, tau hyperphosphorylation, mitochondrial dysfunction, oxidative stress, and acetylcholine deficiency [3]. Although preclinical studies regularly identify promising drug candidates for $\mathrm{AD}$, clinical evidence is still scarce [4]. During the last three decades, targeting the amyloid- $\beta$ (A $\beta$ ) pathway has been central to the search for effective treatments; however, none of the clinical trials targeting $\mathrm{A} \beta$ culmi-

\footnotetext{
${ }^{1}$ Corresponding author; address: pr. Akademika Lavrent'eva 10, Novosibirsk, 630090 Russia; phone: +7(383) 363-49-80; e-mail: kolosova@bionet.nsc.ru.

Abbreviations: AD, Alzheimer's disease; STEP, striatal-enriched protein tyrosine phosphatase; TC-2153, 8-[trifluoromethyl]1,2,3,4,5-benzopentathiepin-6-amine hydrochloride.
}

nated in a significant clinical benefit. Given the complexity of AD pathology and progression, an active search for treatments targeting nonamyloid AD pathologies and for alternative approaches is currently underway. After the finding of elevated striatalenriched tyrosine protein phosphatase (STEP) activity in the prefrontal cortex of human AD patients and in mouse models of AD, modulation of STEP levels or inhibiting its activity are considered one of the promising approaches to the development of a new family of novel therapeutic approaches to this disease $[5,6]$.

The STEP family of protein tyrosine phosphatases includes both cytosolic (STEP46) and membraneassociated (STEP61) members that are formed by alternative splicing of a single gene, PTPN5 [7], which is predominantly expressed in neurons of the basal ganglia, hippocampus, cerebral cortex, and related structures [8]. STEP61 regulates synaptic function and plasticity by regulating $N$-methyl-D-aspartate receptor (NMDAR) and $\alpha$-amino-3-hydroxy-5-methyl-4isoxazolepropionic acid receptor (AMPAR) trafficking (glutamate receptors) as well as ERK1/2, p38, Fyn, and Pyk2 activities. Several pathways, including 
glutamate, dopamine, and brain-derived neurotrophic factor (BDNF) signaling cascades, regulate STEP61 activity, and some of these signaling pathways are implicated in AD. Recent studies indicate that $A \beta$ regulates STEP and that and $A \beta$ accumulation is associated with increases in STEP levels and activity, which in turn disrupts glutamate receptor trafficking to and from the neuronal membrane $[6,9]$. It is believed that STEP opposes the development of longterm potentiation (LTP) and that it imposes a restraint on long-term memory [10]. A $\beta$ enhances long-term depression (LTD) in the hippocampal dentate gyrus region, and the facilitatory effect of $\mathrm{A} \beta$ on LTD involves STEP activation [11]. Thus, it is known that both high and low levels of STEP disrupt synaptic function and contribute to learning and behavioral deficits. The levels and activity of the STEP are increased in the hippocampus of aged memoryimpaired mice and rats, in aged rhesus monkeys, and in people with amnestic mild cognitive impairment; these levels also contribute to the cognitive declines associated with aging [12]. High levels of STEP in addition to $\mathrm{AD}$ are also present in human postmortem samples from patients with Parkinson's disease or schizophrenia as well as in animal models of fragile $\mathrm{X}$ syndrome [13-15]. Low levels of STEP activity are present in other disorders including ischemia, Huntington's chorea, alcohol abuse, and stress disorders [6].

It has been shown that a genetic reduction in STEP levels [16] or pharmacological inhibition of STEP activity (5) can improve memory performance in rodents [12]. $\mathrm{Xu}$ and colleagues [5] have found that a specific STEP inhibitor is 8-(Trifluoromethyl)1,2,3,4,5-benzopentathiepin-6-amine hydrochloride (known as TC-2153), which forms a reversible covalent bond with a catalytic cysteine in STEP. This compound was first synthesized at Novosibirsk Institute of Organic Chemistry (Novosibirsk, Russia) [17] and was designed as an antidepressant by Kulikov and colleagues [18]. As reported [5], brief treatment with TC2153 is effective in reversing cognitive and memory deficits in a transgenic mouse model of AD (3xTg-AD mice) but does not affect $A \beta$ and tau brain pathology of these animals. These results suggest that STEP is a potential target for AD drug discovery. Treatment and prevention of AD may involve prolonged pharmacotherapy; however, the effects of prolonged suppression of STEP with TC-2153 have not been studied previously. Thus, studies of long-term preventive effects of STEP inhibition on cognitive decline in different AD animal models are needed, and above all, in a model of the most prevalent ( $>95 \%$ of cases) sporadic form of the disease.

Here we studied the effects of TC-2153 on the OXYS rat strain: a unique genetic model of accelerated senescence and age-related diseases [19-22]. OXYS rats spontaneously develop all the major signs of $\mathrm{AD}$ and largely reproduce the stages of the disease although there are no mutations specific for familial
$\mathrm{AD}$ in the genome of these rats [19]. Behavioral changes and memory and learning deficits in OXYS rats develop by the age of 3-5 months simultaneously with the first signs of neurodegeneration: disruption of synapses, death of neurons, dysfunction of mitochondria, and hyperphosphorylation of the tau protein in the cerebral cortex and hippocampus. In the OXYS strain, with age, neurodegenerative alterations increase and are accompanied by excessive production of amyloid precursor protein (APP) and accumulation of an $\mathrm{A} \beta$ peptide by the age of 12 months and well-pronounced amyloid-related pathology by the age of 18 months [19, 23]. Recently, we showed that activation of the p38 MAPK signaling pathway accompanies the development of AD-like pathology in OXYS rats [24]. Because STEP regulates this pathway by acting as phosphatase of $\mathrm{p} 38$, we can expect changes in STEP activity. The purpose of this study was to assess the effects of prolonged treatment of OXYS rats with TC2153 on the development of AD signs during their progression: from the age of 9 to 13 months. The behavioral manifestation of $\mathrm{AD}$ and accumulation of $\mathrm{A} \beta$ in the cortex and hippocampus of animals were evaluated. The levels of STEP61 protein were examined too.

\section{MATERIALS AND METHODS}

Animals and treatment. Male senescence-accelerated OXYS rats at the age of 9 months and agematched male Wistar rats (as controls) were obtained from the Breeding Experimental Animal Laboratory of the Institute of Cytology and Genetics, SB RAS (Novosibirsk, Russia, [RFMEFI61914X0005 and RFMEFI61914X0010]; the OXYS strain has been derived from the Wistar strain of rats at the Institute of Cytology and Genetics as described earlier [19, 25]. The rats were kept under conventional conditions on the 12:12 h light/dark cycle (lights on at 8 a.m.) and had ad libitum access to food pellets (PK-120-1; Laboratorsnab, Ltd., Moscow, Russia) and water to 13 months of age. Ethical approval \# 34 from June 15, 2016 was received from the Ethics Committee on Animal Experiments at the Institute of Cytology and Genetics (Novosibirsk, Russia). No animals died during the experiment. Figure 1 shows the design of experiment.

To assess the influence of oral TC-2153 administration (from age 9 to 13 months) on the progression of $\mathrm{AD}$-like pathology, we randomly assigned 9-monthold male OXYS rats to one of two groups (22 rats per group). The experimental groups were set by staff of the Breeding Experimental Animal Laboratory of the Institute of Cytology and Genetics. The size of groups (22 animals in experimental (OXYS+TC-2153), intact (OXYS), and control (Wistar) groups) is optimal for adequate behavioral studies. Previously it was shown that TC-2153 is effective for both enteral (per os) and parenteral (intraperitoneal) administration [5, 17, 18]. TC-2153 was diluted in with $2 \%$ Tween 20 to receive concentration $200 \mathrm{mg} / \mathrm{mL}$ directly before its adminis- 


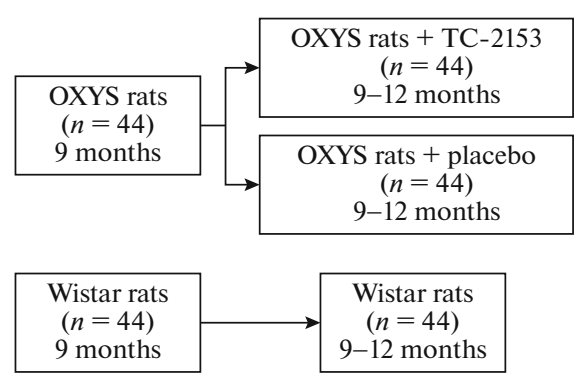

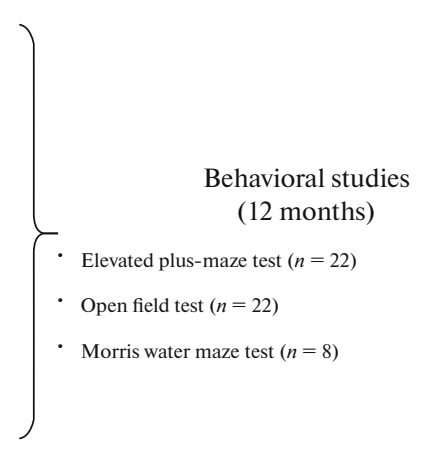

Fig. 1. Experiment design. tration. Than appropriate amount of obtained solution was delivered on the dried bread slice to achieve dosage of $10 \mathrm{mg}$ per $\mathrm{kg}$ of body weight. After that, each rat received one dried bread slice $\left(1 \mathrm{~cm}^{3}\right)$ with TC-2153 individually. Untreated rats received one dried bread slice without TC-2153.

Behavioral testing. Behavioral responses of the animals to treatment with TC-2153 were assessed by several tests in the following order: an anxiety test in an elevated plus maze, testing of locomotor and exploratory activity in the open field test, and learning and memory tasks in the Morris water maze. Behavioral testing was carried out from 12 to 13 months of age. Each rat to be tested was transported from a home cage to the experimental room in a black box. Each test was performed once per animal. Each parameter was rated manually by an observer. The test sessions were scheduled between 10 a.m. and 2 p.m.

The elevated plus maze test. We used this test to quantify anxiety in the untreated OXYS and Wistar rats and in TC-2153-treated OXYS rats $(n=22$ per group). The plus maze apparatus was made of opaque Plexiglas and contained two opposite open arms $(50 \times$ $10 \mathrm{~cm}$ ) and two closed arms of the same size but with 40 -cm-high walls. Each arm was divided by lines into five equal squares $(10 \times 10 \mathrm{~cm})$. The four arms were connected by a central square $\left(100 \mathrm{~cm}^{2}\right)$ and thus formed a plus sign. The apparatus was elevated $50 \mathrm{~cm}$ above the floor. Each rat was placed in the central square of the plus maze, facing one of the closed arms, and its behavior was analyzed for $5 \mathrm{~min}$. We recorded the number of entries into the four arms of the elevated plus maze and the time spent in the arms. A greater amount of time spent in the open arms indicated a reduction of anxiety-like behavior.

The open field test. This test was performed to assess locomotor and exploratory activity of the untreated OXYS and Wistar rats and of TC-2153treated OXYS rats $(n=22$ per group). The animals were subjected to this test at $48 \mathrm{~h}$ after completion of the elevated plus maze test. The open field area consisted of an enclosed square arena made of opaque Plexiglas $(100 \times 100 \mathrm{~cm})$ surrounded by walls $(40 \mathrm{~cm}$ high). The arena was divided by transverse lines into 100 equal squares. A central area was arbitrarily defined as a square of $40 \times 40 \mathrm{~cm}$. A central light source $(100 \mathrm{~W})$ on the ceiling provided invariant illumination in an otherwise dark room. Each rat was placed into the same corner of the arena facing in the same direction and was allowed to freely explore the arena for $5 \mathrm{~min}$. Every time both hind limbs entered a square, a crossing was recorded. The locomotor and exploratory activity was evaluated by counting the line crossings and rearing events (when an animal stood on its hind limbs). In addition, anxiety was evaluated by recording the time before the first entry into the central area. A rat was assumed to be in the central area when its four limbs were on it.

The Morris water maze test of spatial memory. The Morris water maze test [26] was employed to analyze hippocampus-dependent spatial memory by requiring rats ( $n=8$ per group) to find a submerged platform in a pool of water, using external visual cues. Behavioral testing was carried out from 12.5 to 13 months of age as previously described [23]. In brief, the animals were trained in a circular open swimming arena. An escape platform was submerged $2.0 \mathrm{~cm}$ below the surface of the water, which was mixed with powdered milk to obscure the platform. Two principal axes of the maze were designated dividing the arena into four equal quadrants. The platform was positioned in the middle of the first quadrant throughout the 5-day training period. Trials (five consecutive days with four trials per day) were conducted with the same hidden platform location using a semirandom set of start locations; one trial each day was initiated from each of the four positions. Each trial either lasted for $70 \mathrm{~s}$ or ended when a rat reached the submerged platform, thus escaping water. Reference memory was quantified using a preference for the platform area when the platform was absent in the probe trial. Each trial was recorded by a camera, than ciphered and rated manually by an observer.

Tissue preparation. At the end of the animal behavioral testing, TC-2153-treated OXYS rats and untreated OXYS and Wistar rats were euthanized by $\mathrm{CO} 2$ asphyxiation and decapitation. For an enzyme- 
linked immunosorbent assay (ELISA) and western blotting, the hippocampus and prefrontal cortex were quickly separated from the left hemisphere, placed in microcentrifuge tubes for protein isolation, and frozen in liquid nitrogen. The tissues were stored at $-70^{\circ} \mathrm{C}$ until analysis.

Frozen tissues of the hippocampus and prefrontal cortex of the TC-2153-treated and untreated OXYS and Wistar rats ( $n=6$ to 8 per group) were homogenized in protein lysis buffer RIPA ( $50 \mathrm{mM}$ Tris- $\mathrm{HCl}$ $\mathrm{pH} 7.4,150 \mathrm{mM} \mathrm{NaCl}, 1 \%$ Triton $\mathrm{X}-100,1 \%$ sodium deoxycholate, $0.1 \%$ SDS, $1 \mathrm{mM}$ EDTA) supplemented with a protease inhibitor cocktail (cat. \# P8340, Sigma-Aldrich, St. Louis, MO, USA). After incubation for $20 \mathrm{~min}$ on ice, the samples were centrifuged at $12000 \mathrm{~g}$ for $30 \mathrm{~min}$ at $4^{\circ} \mathrm{C}$, and the supernatants were transferred to new tubes. Total protein was quantified using the Bio-Rad Bradford Kit (Bio-Rad Laboratories, Hercules, CA, USA).

ELISAs. An ELISA for amyloid- $\beta 1-42$ (Human/Rat ELISA Kit; Wako, Japan) was performed on the isolated protein samples according to the manufacturer's instructions. Quantitation was carried out based on measurement of optical density on a microtiter plate reader, and the concentration was calculated in picograms of the $A \beta 1-42$ peptide per milligram of total protein of hippocampal or brain cortical tissue.

Western blotting. Proteins (30 $\mu \mathrm{g}$ per lane) were resolved by electrophoresis on an SDS $10 \%$ polyacrylamide gel and electrotransferred onto nitrocellulose membranes. After blocking for $2 \mathrm{~h}$ with a solution of $5 \%$ bovine serum albumin (BSA; cat. \# SLBJ8588V, Sigma-Aldrich, St. Louis, MO, USA) in PBS containing $0.1 \%$ of Tween 20 , each membrane was probed with a polyclonal mouse anti-STEP antibody (dilution 1 : 1000; cat. \# sc-2031, Santa Cruz Biotechnology, Dallas, TX, USA) overnight at $4^{\circ} \mathrm{C}$. Then, there was an immunoreaction with a corresponding secondary antibody (dilution 1: 5000; cat. \# ab6808; Abcam, Cambridge, MA, USA) for $2 \mathrm{~h}$ at room temperature. The blots were treated with the SuperSignal West Femto Maximum Sensitivity Substrate (Thermo Fisher Scientific Inc., Waltham, MA, USA) according to the manufacturer's instructions. Band intensities were quantitated in the ImageJ software (NIH, Bethesda, MD, USA). For reference protein staining, we chose an anti- $\beta$-actin antibody (dilution $1: 5000$; cat. \# ab1801, Abcam, Cambridge, MA, USA).

Statistical analysis. The data were analyzed by oneway and two-way ANOVA (Statistica 8.0 software; StatSoft, Tulsa, OK, USA). The normality of distribution was examined using Shapiro-Wilk criterion. The Newman-Keuls post hoc test was applied to significant main effects and interactions in order to assess the differences between untreated OXYS rats and Wistar rats as well as between untreated and treated OXYS rats. The $t$ - test for dependent samples was performed for dependent-pair comparison. The data are presented as mean \pm SEM. The differences were considered statistically significant at $p<0.05$.

\section{RESULTS}

Assessment of animal behavior in the elevated plus maze test. In this study, the Newman-Keuls post hoc test did not detect any differences in the anxiety level between OXYS and Wistar rats in the elevated plus maze test (Figs. 2a-2d): indeed, the number of entries into open arms and the time spent and the distance passed in it did not significantly differ between the strains $(p>0.05)$. At the same time, TC-2153 treatment decreased the time spent in open arms of the maze by OXYS rats $(p<0.02$; the Newman-Keuls test), thus causing an increase in anxiety; however, the parameter did not differ between TC-2153-treated OXYS rats and control Wistar rats.

Locomotor activity was significantly lower in OXYS rats: the distance passed in closed arms of the maze and the number of rearings were lower than those in Wistar rats $(p<0.008$ and $p<0.006$, respectively; the Newman-Keuls test). Treatment with TC2153 did not affect these parameters in OXYS rats. According to the the Newman-Keuls test exploratory activity-which means stretched attend postures and head dips-did not differ between OXYS and Wistar rats, and TC-2153 treatment did not influence these parameters.

Thus, we found no differences in the anxiety level between OXYS and Wistar rats (Figs. 1e, 1f); however, the TC-2153 treatment may increase anxiety in OXYS rats. As for locomotor activity, it was low in OXYS rats and was not affected by TC-2153 treatment.

\section{Animal Behavior in the Open Field Test}

The results of the behavioral observations in the open field test are presented in Fig. 3. As expected, ANOVA revealed that OXYS rats were significantly more passive than age-matched Wistar rats: the number of squares crossed and frequency of rearings were significantly lower in OXYS rats $\left(\mathrm{F}_{1,45}=13.4, p<0.001\right.$ and $\mathrm{F}_{1,45}=10.0, p<0.003$, respectively).

The time of the first entry into the central area of the open field apparatus is associated with less anxiety. Latency to enter the central area of the open field was longer in OXYS rats when compared with Wistar rats $\left(\mathrm{F}_{1,45}=5.7, p<0.02\right.$ according to ANOVA $)$ which may reflect increased anxiety of OXYS rats. Lower frequency of grooming behavior may be associated with lower anxiety and/or decreased activity. ANOVA showed that grooming frequency was slightly lower in OXYS rats $\left(\mathrm{F}_{1,45}=3.9, p=0.05\right)$ compared to Wistar rats.

TC-2153 treatment significantly decreased exploratory and locomotor behavior of OXYS rats in the 
(a)

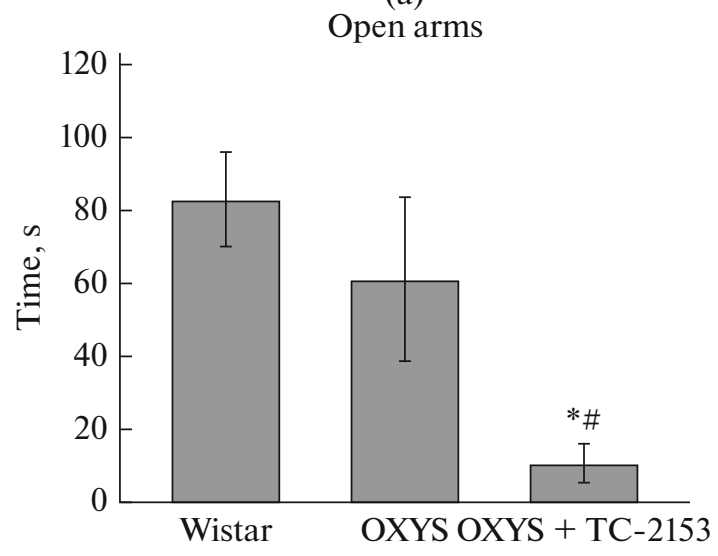

(c)

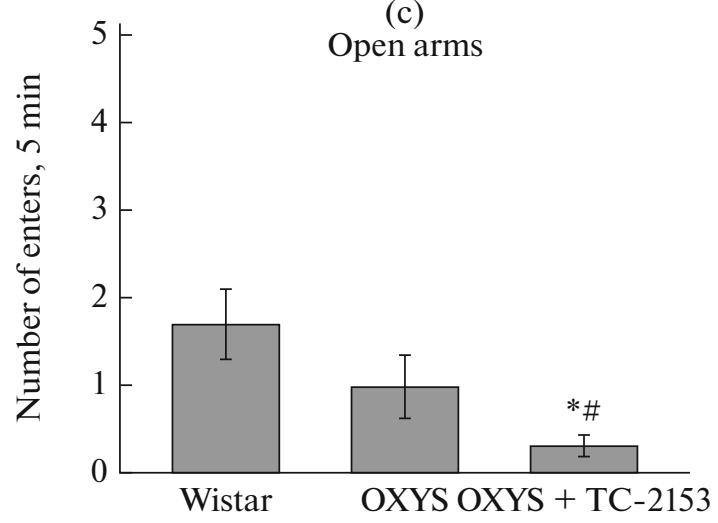

(e)

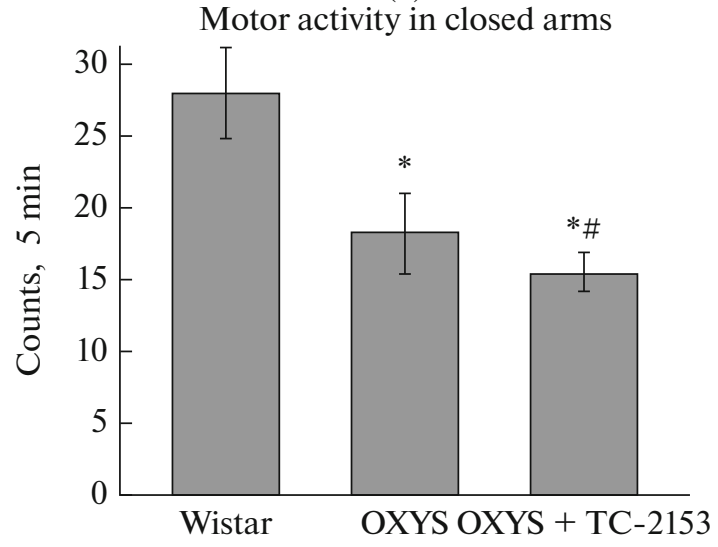

(b)

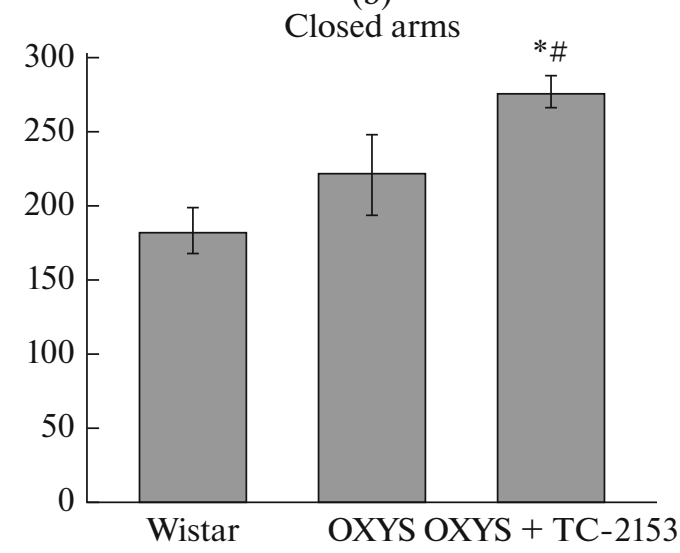

(d)

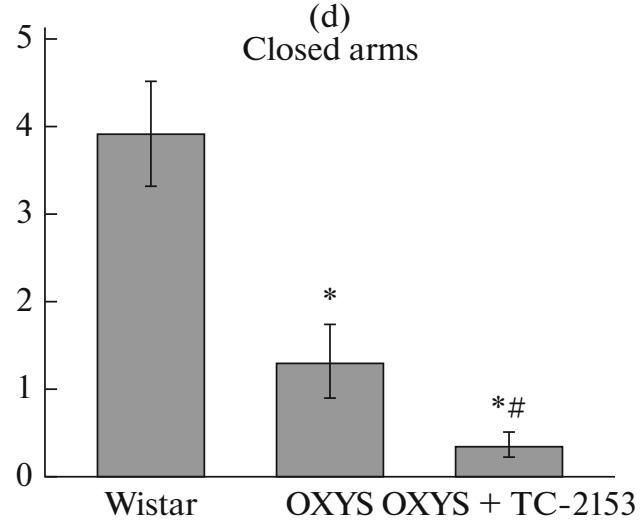

(f)

Number of rearings

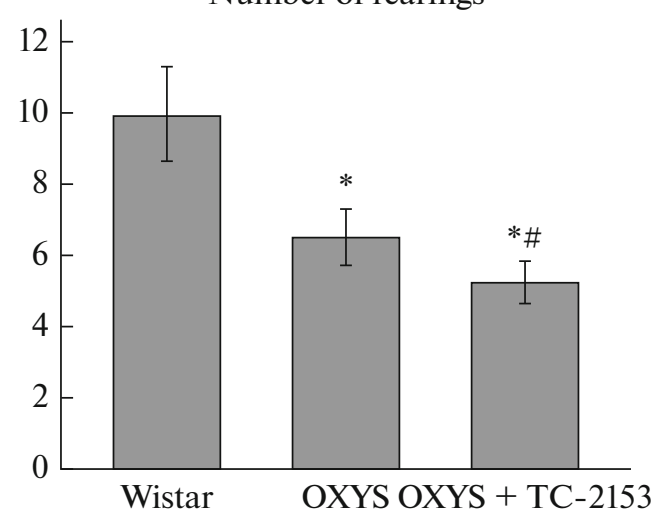

Fig. 2. The effects of TC-2153 treatment on the performance of OXYS rats in the elevated plus maze test. OXYS rats made fewer entries into the closed arms (d) as compared to Wistar rats. Oral administration of TC-2153 decreased the time spent in the open arms (a) and the number of the entries in both open (c) and closed (d) arms by OXYS rats. OXYS rats showed a significantly lower frequency of rearings (f) when compared with Wistar rats. TC-2153 significantly decreased the number of squares crossed (e) and frequency of rearings (f) in OXYS rats. The data are shown as mean $\pm \mathrm{SEM} ;{ }^{*} p<0.05$ for differences between the strains; ${ }^{\#} p<$ 0.05 for effects of TC-2153.

open field test-i.e., a decreased number of squares crossed and frequency of rearings $\left(\mathrm{F}_{1,36}=4.1, p<0.05\right.$ and $\mathrm{F}_{1,37}=5.1, p<0.03$, respectively according to ANOVA) - and had no effect on the latency to enter the central area of the open field and on grooming frequency.
Examination of the rats' learning ability and reference memory in the Morris water maze test. ANOVA showed only slight effect of genotype $\left(\mathrm{F}_{1,109}=3.5, p=\right.$ 0.064 ) on latency period of finding the platform; and post hoc analysis did not reveal differences in the learning ability between OXYS and Wistar rats (Fig. 4a): animals 
(a)

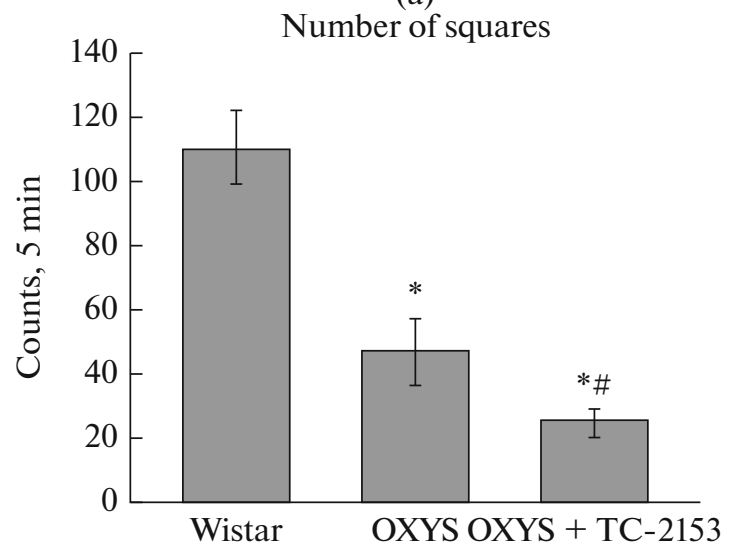

(b)

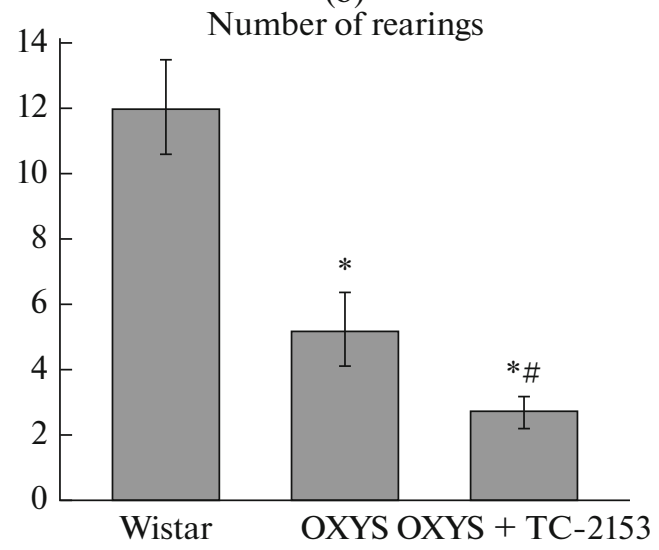

Fig. 3. The effects of TC-2153 treatment on the behavior of OXYS rats in the open field test. OXYS rats showed a significantly lower number of squares crossed (a) and frequency of rearings (b) as compared to Wistar rats. TC-2153 significantly decreased the number of squares crossed (a) and frequency of rearings (b) in OXYS rats. The data are shown as mean \pm SEM; ${ }^{*} p<0.05$ for differences between the strains; $\# p<0.05$ for effects of TC-2153; $n=22$ in group.

(a)

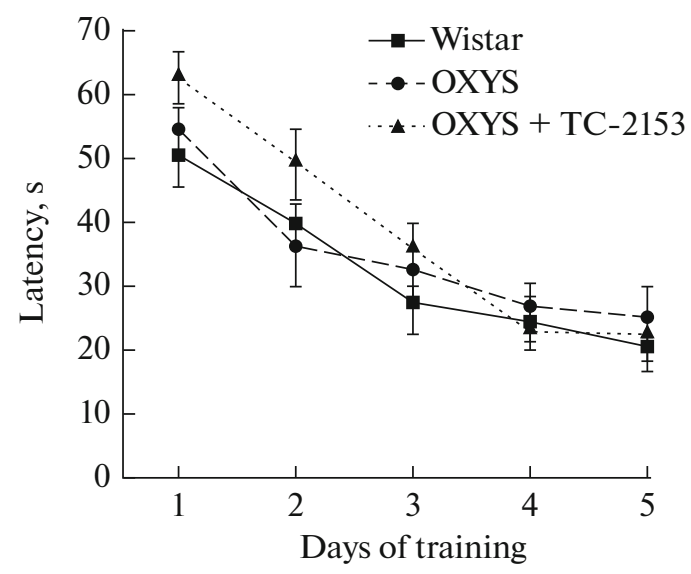

(b)

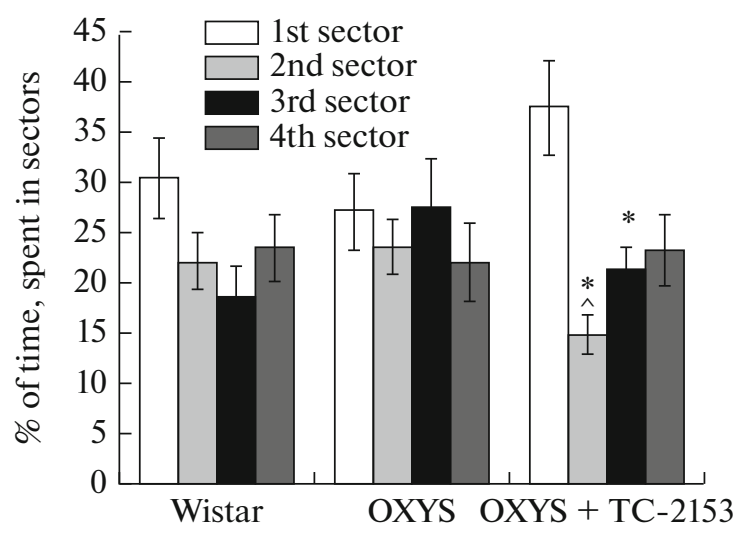

Fig. 4. Effects of TC-2153 on spatial memory of OXYS rats in the Morris water maze test. The latency of finding the platform (a) decreased from the 1st to 5th day of training. TC-2153 increased time spent by OXYS rats in target sector 1 compared to other sectors (b) on the 6th day of training when the platform was removed. Swimming speed (a) did not differ between animal groups. The data are depicted as mean \pm SEM; Legend: ${ }^{\wedge} p<0.05$ for differences with a previous training day; ${ }^{\circledR} p<0.05$ for differences between target sector 1 and other sectors in the time spent there. The black line denotes average percentage of time which should be spent by untrained animals in all sectors; $n=8$ in group.

of both strains learned to find a platform hidden under water in sector 1 already on the 2 nd day of testing $(p<$ 0.05 ; the $t$-test for dependent samples). Naturally, the latency period of finding the platform gradually diminished by the 5th day of training in rats of both strains $\left(\mathrm{F}_{4,109}=26.0, p<0.0001\right.$ according to ANOVA), thus revealing the effect of testing day. OXYS rats receiving TC-2153 also learned to find a hidden platform on the 2 nd day of training $(p<0.006$; the $t$-test for dependent samples), whereas the latency period of finding the platform significantly decreased by the 3 rd day of training ( $p<0.05$; the $t$-test for dependent samples) and continued to decrease gradually to the 5th training day.

NEUROCHEMICAL JOURNAL Vol. 15 No. 32021
Reference memory was evaluated on the 6th day, when the platform was removed, by the $t$-test for dependent samples. ANOVA did not reveal any interstrain differences in time spent by the animals in the target sector 1 on 6 th day of testing $\left(\mathrm{F}_{1,22}=0.1, p=\right.$ $0.74)$; however, within the strain Wistar rats demonstrated a preference for target sector $1(p<0.04)$, which indicates that the animals remembered the location of the platform (Fig. 4b). OXYS rats, in contrast, spent approximately the same time in all four sectors $(p=0.58)$. TC-2153 administration had a beneficial effect on long-term memory of the animals: OXYS rats receiving the drug spent significantly more time in target sector $11(p<0.02)$. 
(a)
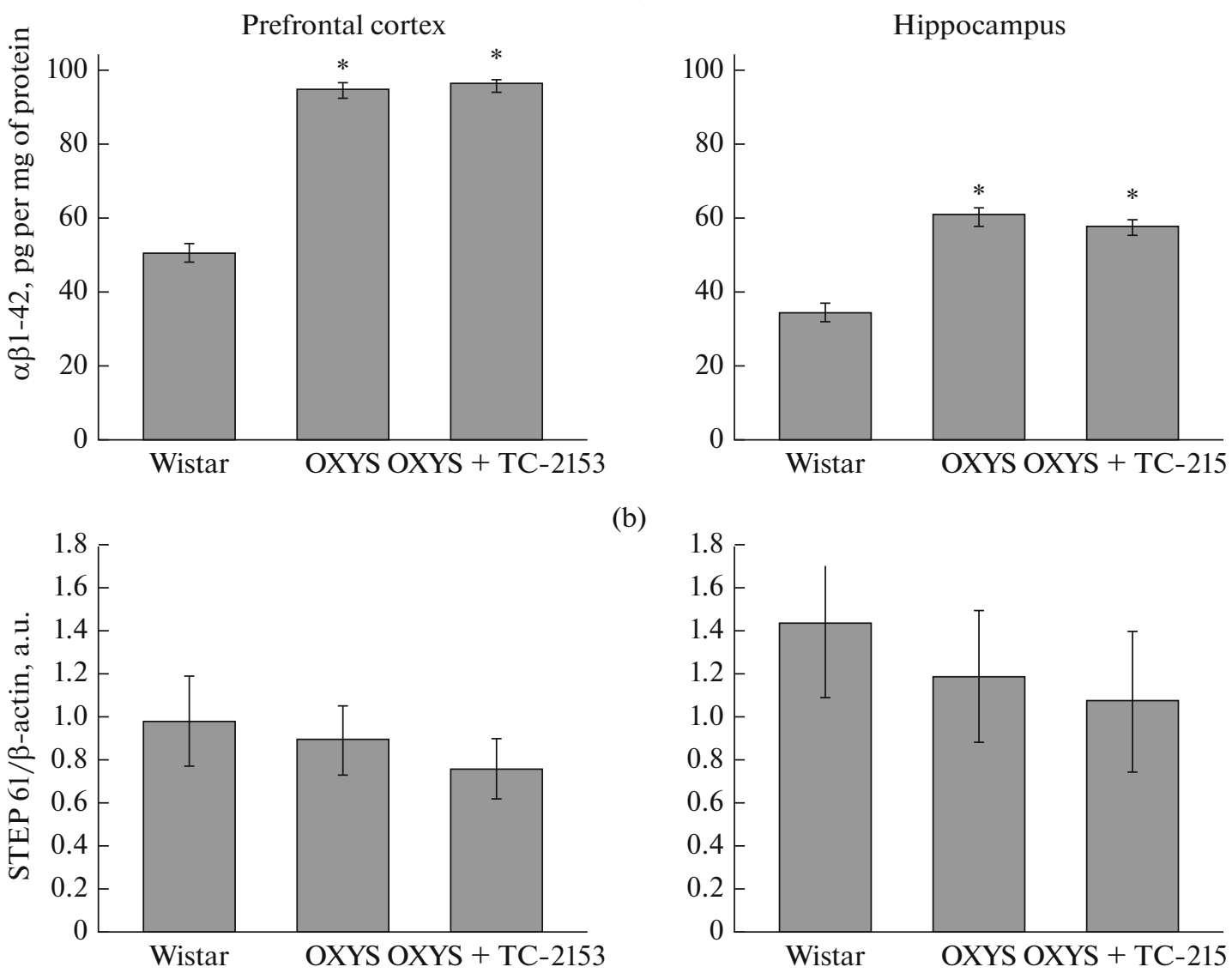

(b)
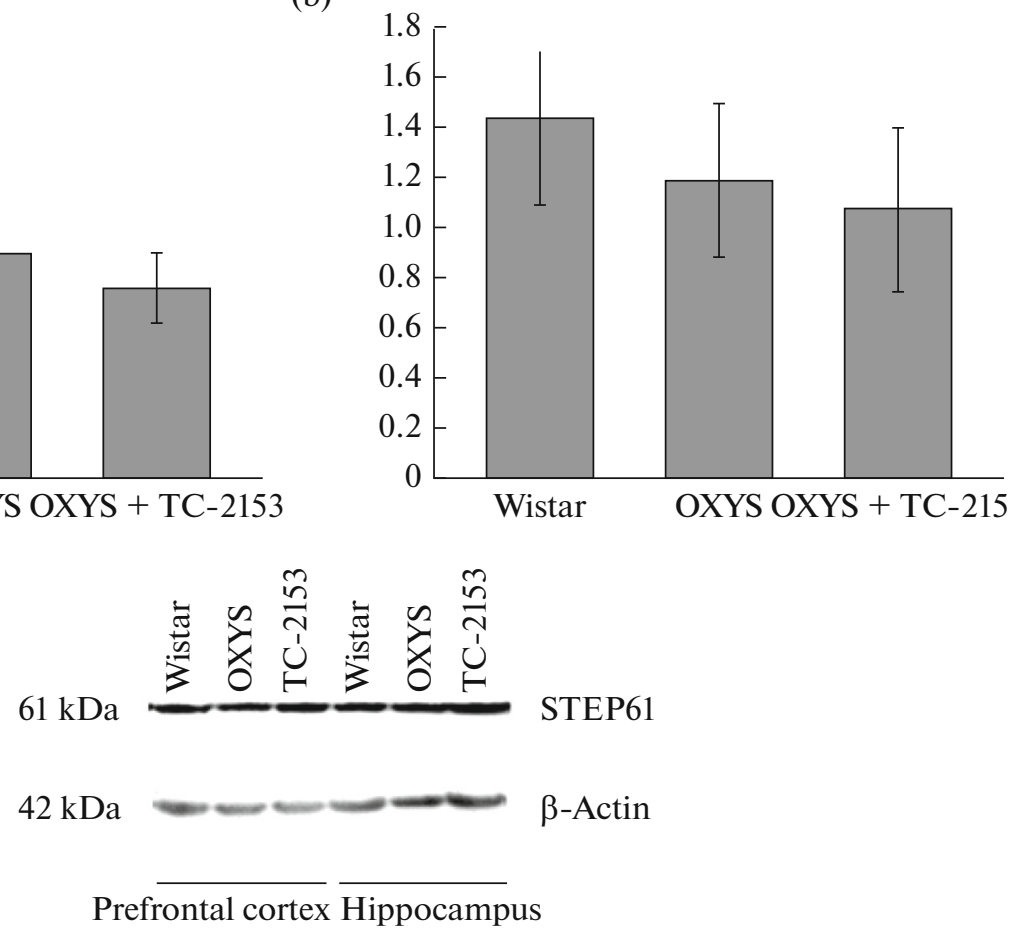

Fig. 5. Protein levels of A $\beta 1-42$ and of the STEP61 isoform in the cortex and hippocampus of control Wistar and OXYS rats and TC-2153-treated OXYS rats. The A $\beta 1-42$ levels (a) were higher in the cortex and hippocampus of OXYS rats. The levels of STEP61 (b) did not differ between OXYS and Wistar rats in the cortex and hippocampus. TC-2153 treatment did not affect either protein's level. The data are given as mean $\pm \mathrm{SEM} ;{ }^{*} p<0.05$ for differences between the strains.

It's important to point out, that swimming speed (Fig. 4c) did not significantly differ between OXYS and Wistar rats at all training days, and TC-2153 treatment did not affect it.

Effects of TC-2153 on A $\beta$ levels in the hippocampus and frontal cortex. In this study, we tested whether treatment with TC-2153 since 9 months of age affects $\mathrm{A} \beta 1-42$ content of the hippocampus and frontal cortex of OXYS rats. ANOVA showed that the amounts of $A \beta 1-42$ in the hippocampus and frontal cortex of OXYS rats were significant higher than those in Wistar rats $\left(\mathrm{F}_{1,18}=146, p<0.0001\right.$, and $\mathrm{F}_{1,18}=86, p<0.0001$, respectively) and were not affected by TC-2153 $\left(\mathrm{F}_{1,18}=\right.$ $0.38, p=0,54$, and $\mathrm{F}_{1,18}=0.88, p=0.36$, respectively). The level of $A \beta 1-42$ in the cerebral cortex and in the hippocampus of OXYS rats remained approximately twice as high as that of Wistar rats (Fig. 5a).

Effects of TC-2153 on STEP61 protein levels in the cerebral cortex and hippocampus of OXYS rats. Next, we investigated the levels of STEP61 isoform in the cortex and hippocampus by Western blot analysis (Fig. 5b). At the age of 13 months, ANOVA showed that the difference between the genotypes (strains) disappeared for the cortex $\left(\mathrm{F}_{1,8}=0.12, p=0.73\right)$ and hippocampus 
$\left(\mathrm{F}_{1,10}=0.31, p=0.59\right)$. Supplementation with TC2153 had no effect on the STEP61 protein level in either brain structure of OXYS rats $\left(\mathrm{F}_{1,8}=0.39, p=\right.$ 0.55 , and $\mathrm{F}_{1,10}=0.057, p=0.55$, respectively according to ANOVA).

\section{DISCUSSION}

The prolonged supplementation with STEP inhibitor TC-2153 reduced locomotor and exploratory activities in the open field test by twofold and significantly increased anxiety in the elevated plus maze test when compared with untreated OXYS rats. In OXYS rats behavioral alterations start by the age of 3 months, progressively increase with age, and as confirmed by our present study, at the age of 13 months, the behaviors of OXYS and Wistar rats significantly differ against the background of gradual accumulation of $\mathrm{A} \beta$. Thus, TC-2153 adversely affected behavioral manifestations associated with the development of AD-like pathology. At the same time, we did not reveal negative effects of TC-2153 on the learning of OXYS rats in the Morris water maze test. It should be noted that spatial learning of OXYS rats at 13 months of age does not significantly differ from that of Wistar rats. OXYS rats demonstrate significant deterioration of cognitive function in the Morris water maze test starting from age 14-16 months [27, 28]. In contrast, OXYS rats manifest learning and reference memory deficits in the 8-arm radial maze already at 3 months of age [29]. Moreover, TC-2153 had a beneficial effect on long-term memory of the animals: OXYS rats receiving the drug spent significantly more time in target sector 1 . As reported earlier, short-term inhibition of STEP by TS-2153 has beneficial effects on hippocampus-dependent memory: indeed, it improves agerelated memory deficits of old Sprague-Dawley rats (20-22 months of age) trained in the T-maze memory task [12] as well as reverses cognitive deficits in 12-month-old 3xTg-AD mice [5]. It is well known that performance on the Morris water maze test is linked to long-term potentiation and NMDA receptor function, and this test is a key technique for the investigation of hippocampal circuitry [30, 31]. In our study, we did not notice alterations of the learning ability of OXYS rats in the Morris water maze test. Thus, TC-2153 did not affect the learning ability. Nonetheless, reference memory was found to be worse in OXYS rats, whereas long-term treatment with TC-2153 improved it. Therefore, in this regard, our data match the results about the effects short-term TC-2153 administration on cognitive performance [5]. They are also consistent with the observed absence of an impact of TC-2153 on the level of A $\beta$. Besides, the short-term inhibition of STEP by TC-2153 does not significantly change $A \beta$ or phospho-tau levels in 6-month-old 3xTg-AD mice [5]; the prolonged administration of TC-2153 in our study also did not affect the level of $A \beta$ in the hippocampus and prefrontal cortex of OXYS rats. Impor- tantly, in their last publication Chatterjee et al. [32] report that ability of TC-2153 to improve cognitive function in the $3 \times \mathrm{Tg}-\mathrm{AD}$ mice is associated with increasing of in synaptic connectivity.

However, not only functional, but also silent synapses may be formed during synaptogenesis [33, 34]. Previously we have showed increased synaptogenesis in OXYS rats at 5 months of age; however, newly generated synapses were dysfunctional [20]. Thus, generation of silent synapses in the hippocampus under TC2153 treatment may underlie observed decreased locomotion and increased anxiety in OXYS rats; this possibility should be further investigated.

We did not detect any differences in the level of the STEP61 protein: it was the same between Wistar and OXYS rats and was not affected by the prolonged administration of TC-2153. According to Jian Xu and co-workers [5], TC-2153 inhibits STEP activity by forming a covalent bond with active-site Cys472, which is required for the enzymatic activity, without changing the amount of total STEP and phosphorylated STEP. In the present study, we did not examine the activity and phosphorylation of STEP61 but evaluated them when investigating the effects of TC-2153 on the retina of OXYS rats [35]. AD-like pathology develops in OXYS rats simultaneously with other manifestations of accelerated senescence, one of which is retinopathy, similar to age-related macular degeneration (AMD) in humans [21]. This disorder shares several clinical and histopathological features with $\mathrm{AD}$, and some researchers regard $\mathrm{AMD}$ as $\mathrm{AD}$ of the eye [36]. Recently, we showed [35] that an increase in the STEP61 protein level and a decrease in total and STEP phosphatase activities in the retina (as compared with Wistar rats) precede the manifestation of clinical signs of AMD in OXYS rats (age 20 days). At the same time, we did not note any differences in these retinal parameters between 13-month-old Wistar rats and OXYS rats (the latter show pronounced signs of AMD). Moreover, inhibition of STEP with TC-2153 at the same age and duration as in the present study (from 9 to 13 months of age) has a negative effect on the neuroretina while having no influence on Ptpn5 mRNA expression, STEP46 and STEP61 protein levels, and their phosphatase activity in the OXYS retina [35]. Those results, just as the data obtained in the present study, suggest that the effects of prolonged treatment with TC-2153 may be mediated by mechanisms not related to STEP. Previously, it has been demonstrated [9] that effects of TC-2153 could be mediated by its influence on both the brain serotoninergic system and BDNF, whose age-related alterations are involved in the development of neurodegenerative disorders. Indeed, previously, we have shown almost a twofold decrease in the BDNF level in the hippocampus of OXYS rats compared with Wistar rats at 12 months of age [29]. In addition, it was found that STEPs are redox-sensitive enzymes: on the one hand, oxidative stress induced by hydrogen peroxide leads to 
a significant increase in the formation of dimers and higher-order oligomers of STEP61 thereby causing a significant reduction in its enzymatic activity [37]; on the other hand, the inhibition of STEP by TC-2153 can be reversed by incubation with thiols such as glutathione or dithiothreitol [5]. A recent review by Gü Gojon and G.A. Morales [38] discusses the prospects of using TC-2153 and others catenated divalent organic sulfur compounds as promising hydrogen sulfide prodrugs that can be considered potential hydrogen sulfide $\left(\mathrm{H}_{2} \mathrm{~S}\right)$ donors. $\mathrm{H}_{2} \mathrm{~S}$ is an endogenous gasotransmitter that is capable of modulating the cell's redox homeostasis by acting as an antioxidant and featuring a wide variety of cytoprotective and physiological functions in age-associated diseases [39]. It is noteworthy that a significant decline of $\mathrm{H}_{2} \mathrm{~S}$ levels has been observed in AD patients, suggesting that the use of $\mathrm{H}_{2} \mathrm{~S}$ donors is a promising strategy for the treatment of neurodegenerative diseases [40]. Indeed, the effects of short-term exposure to TC-2153 represent positive results in a transgenic model of $\mathrm{AD}$, depressive disorders [9], and autism [41]. However, it should be noted that not only oxidative but also reductive stress such as excess of the $\mathrm{H} 2 \mathrm{~S}$ takes place in the cell and may lead to adverse effects [42]. The findings in our study of prolonged TC-2153 administration on the development of both AD- and AMD-like pathology in OXYS rats are contradictory. Indeed, prolonged administration of TC-2153 here improved long-term memory of OXYS rats while significantly reducing the already decreased locomotor and exploratory activities and increasing their anxiety: the alterations that we consider a manifestation of accelerated senescence of these rats' brain. An even more alarming result of long-term treatment with TC-2153 may be its negative influence on retinal neurons, as we reported previously [35]. Thus, prolonged administration of TC2153 may exert longitudinal actions on redox status by altering an oxidation and reduction balance. Excess reduction potential may affect cellular signaling pathways, modify transcriptional activity, induce alterations in the formation of disulfide bonds in proteins, reduce mitochondrial function, and decrease cellular metabolism [43]. It is no coincidence that excess consumption of dietary antioxidant supplements, such as vitamins and/or flavonoids, may have pro-oxidant effects that may alter the redox cellular equilibrium and contribute to reductive stress, thereby even diminishing life expectancy.

\section{CONCLUSION}

We agree with Gü Gojon and G.A. Morales [38] that TC-2153 as well as its analogues have a potential to become effective pharmacological therapeutic agents. Nevertheless, additional studies are needed to investigate the mechanisms of TC-2153 action and to find optimal doses and duration of administration in various animal models.

\section{ACKNOWLEDGMENTS}

The animals were kindly provided by the Breeding Experimental Animal Laboratory of the Institute of Cytology and Genetics, SB RAS (Novosibirsk, Russia). The English language was corrected and certified by shevchukediting.com.

\section{FUNDING}

This work was supported by the Russian Ministry of Science and Higher Education within grant number 14.W03.31.0034 (mega-grant). The sponsors had no role in study design; in the collection, analysis, and interpretation of data; in the writing of the report; or in the decision to submit the article for publication.

\section{COMPLIANCE WITH ETHICAL STANDARDS}

Conflict of interest. The authors declare no conflict of interest.

Ethical approval. All applicable international, national, and/or institutional guidelines for the care and use of animals were followed.

Author contributions. Conceptualization N.G.K.; Investigation, A.O.B., T.A.K., D.V.T., N.A.M. and E.A.R; T.M.K., K.P.V. and N.F.S. synthesized the inhibitor of STEP (TC2153); Writing-original draft, D.V.T., N.G.K. and E.A.R. All authors have read and agreed to the published version of the manuscript.

\section{OPEN ACCESS}

This article is licensed under a Creative Commons Attribution 4.0 International License, which permits use, sharing, adaptation, distribution and reproduction in any medium or format, as long as you give appropriate credit to the original author(s) and the source, provide a link to the Creative Commons license, and indicate if changes were made. The images or other third party material in this article are included in the article's Creative Commons license, unless indicated otherwise in a credit line to the material. If material is not included in the article's Creative Commons license and your intended use is not permitted by statutory regulation or exceeds the permitted use, you will need to obtain permission directly from the copyright holder. To view a copy of this license, visit http://creativecommons.org/licenses/by/4.0/.

\section{REFERENCES}

1. Frere, S. and Slutsky, I., Neuron, 2018, vol. 97, pp. 3258.

2. Alzheimer's Disease International. World Alzheimer Report 2019: Attitudes to Dementia. Alzheimer's Disease International: London, UK, 2019, pp. 13-16

3. Querfurth, H.W. and LaFerla, F.M., N. Engl. J. Med. 2010, vol. 362, pp. 329-344. 
4. Crous-Bou, M., Minguillón, C., Gramunt, N., and Molinuevo, J.L., Alzheimers Res. Ther., 2017, vol. 9, p. 71 .

5. Xu, J., Chatterjee, M., Baguley, T.D., Brouillette, J., Kurup, P., Ghosh, D., et al., PLoS Biol., 2014, vol. 12, p. 1001923 .

6. Kamceva, M., Benedict, J., Nairn, A.C., and Lombroso, P.J., Neural. Plast., 2016, vol. 2016, p. 8136925.

7. Bult, A., Zhao, F., Dirkx, R., Jr., Sharma, E., Lukacsi, E., Solimena, M., et al., J. Neurosci., 1996, vol. 16, pp. 7821-7831.

8. Boulanger, L.M., Lombroso, P.J., Raghunathan, A., During, M.J., Wahle, P., and Naegele, J.R., J. Neurosci., 1995, vol. 15, pp. 1532-1544.

9. Kulikova, E. and Kulikov, A., Curr. Protein Pept. Sci., 2017, vol. 18, pp. 1152-1162.

10. Saavedra, A., Ballesteros, J.J., Tyebji, S., MartínezTorres, S., Blázquez, G., López-Hidalgo, R., et al., Mol. Neurobiol., 2019, vol. 56, pp. 1475-1487.

11. Chen, X., Lin, R., Chang, L., Xu, S., Wei, X., Zhang, J., et al., Neuroscience, 2013, vol. 253, pp. 435-443.

12. Castonguay, D., Dufort-Gervais, J., Ménard, C., Chatterjee, M., Quirion, R., Bontempi, B., et al., Curr. Biol., 2018, vol. 28, pp. 1079-1089. e4.

13. Carty, N. C., Xu, J., Kurup P., Brouillette, J., GoebelGoody, S.M., Austin, D.R., et al., Transl. Psychiatry, 2012, vol. 2, article e137.

14. Goebel-Goody, S.M., Wilson-Wallis, E.D., Royston, S., Tagliatela, S.M., Naegele, J.R., and Lombroso, P.J., Genes Brain Behav., 2012, vol. 11, pp. 586-600.

15. Kurup, P.K., Xu, J., Videira, R.A., Ononenyi, C., Baltazar, G., Lombroso, P.J., and Nairnc, A.C., Proc. Natl. Acad. Sci. USA. 2015, vol. 112, pp. 1202-1207.

16. Zhang, Y., Kurup, P., Xu, J., Carty, N., Fernandez, S.M., Nygaard, H.B., et al., Proc. Natl. Acad. Sci. USA, 2010, vol. 107, pp. 19014-19019.

17. Khomenko, T.M., Tolstikova, T.G., Bolkunov, A.V., Dolgikh, M.P., Pavlova, A.V., Korchagina, D.V., et al., Lett. Drug. Des. Discov., 2009, vol. 6, pp. 464-467.

18. Kulikov, A.V., Tikhonova, M.A., Kulikova, E.A., Volcho, K.P., Khomenko, T.M., Salakhutdinov, N.F., et al., Psychopharmacology (Berl.), 2012. vol. 221, pp. 469-478.

19. Stefanova, N.A., Kozhevnikova, O.S., Vitovtov, A.O., Maksimova, K.Y., Logvinov, S.V., Rudnitskaya, E.A., et al., Cell Cycle, 2014, vol. 13, pp. 898-909.

20. Stefanova, N.A., Muraleva, N.A., Korbolina, E.E., Kiseleva, E., Maksimova, K.Y., and Kolosova, N.G., Oncotarget, 2015, vol. 6, pp. 1396-1413.

21. Telegina, D.V., Kozhevnikova, O.S., Bayborodin, S.I., and Kolosova, N.G., Sci. Rep., 2017, vol. 7, pp. 41533.
22. Telegina, D.V., Suvorov, G.K., Kozhevnikova, O.S., and Kolosova, N.G., Int. J. Mol. Sci., 2019, vol. 20, p. 5632.

23. Stefanova, N.A., Muraleva, N.A., Skulachev, V.P., and Kolosova, N.G., J. Alzheimers Dis., 2014, vol. 38, pp. 681-694.

24. Muraleva, N.A., Stefanova, N.A. and Kolosova, N.G., Antioxidants (Basel), 2020, vol. 9, p. 676.

25. Soloveva, N.A., Morozkova, T.S. and Salganik, R.I., Genetika, 1975, vol. 11, pp. 63-71.

26. Morris, R. J. Neurosci. Methods, 1984, vol. 11, pp. 4760.

27. Stefanova, N.A., Fursova, A.Z., and Kolosova, N.G., J. Alzheimers Dis., 2010, vol. 21, pp. 479-491.

28. Stepanichev, M.Y., Onufriev, M.V., Peregud, D.I., Lazareva, N.A., Moiseeva, Y.V., Nesterenko, A.N., et al., Neurochem. J., 2018, vol. 12, pp. 184-194.

29. Rudnitskaya, E.A., Maksimova, K.Yi., Muraleva, N.A., Logvinov, S.V., Yanshole, L.V., Kolosova, N.G., et al., Biogerontology, 2015, vol. 16, pp. 303-316.

30. Vorhees, C.V. and Williams, M.T., Nat. Protoc., 2006, vol. 1, pp. 848-858.

31. Nunez, J. J. Vis. Exp., 2008, vol. 19, p. 897.

32. Chatterjee, M., Kwon, J., Benedict, J., Kamceva, M., Kurup, P., and Lombroso, P.J., Exp. Brain Res., 2021. https://doi.org/10.1007/s00221-020-06028-X

33. Kullmann, D.M., Philos. Trans. R. Soc. Lond. B. Biol. Sci., 2003, vol. 358, pp. 727-733.

34. Koya, E., Dong, Y. Neuropsychopharmacology, 2018, vol. 43, pp. 1981-1982.

35. Telegina, D.V., Kulikova, E.A., Kozhevnikova, O.S., Kulikov, A.V., Khomenko, T.M., Volcho, K.P., et al., Int. J. Mol. Sci., 2020, vol. 21, pp. 5182.

36. Papadopoulos, Z., Curr. Aging Sci., 2020, vol. 13, pp. 102-112.

37. Deb, I., Poddar, R., and Paul, S., J. Neurochem., 2011, vol. 116, pp. 1097-1111.

38. Gojon, G. and Morales, G.A., Antioxid. Redox Signal, 2020, vol. 33, pp. 1010-1045.

39. Tabassum, R., Jeong, N.Y. and Jung, J., Neural Regen. Res., 2020, vol. 15, pp. 653-662.

40. Sestito, S., Pruccoli, L., Runfola, M., Citi, V., Martelli, A., Saccomanni, G., et al., Eur. J. Med. Chem., 2019, vol. 184, p. 111745 .

41. Chatterjee, M., Singh, P., Xu, J., Lombroso, P.J., and Kurup, P.K., Behav. Brain. Res., 2020, vol. 391, p. 112713.

42. Lloret, A., Fuchsberger, T., Giraldo, E., and Vina, J., Curr. Alzheimer Res., 2016, vol. 13, pp. 206-211.

43. Pérez-Torres, I., Guarner-Lans, V., and Rubio-Ruiz, M.E., Int. J. Mol. Sci., 2017, vol. 18, p. 2098. 